\title{
Nasal Cavity and Paranasal Sinus Carcinoma
}

National Cancer Institute

\section{Source}

National Cancer Institute. Nasal Cavity and Paranasal Sinus Carcinoma. NCI Thesaurus.

Code C54293.

A carcinoma arising from the nasal cavity or paranasal sinuses. 